\title{
Fabrication of Bio-Functionalised Polypyrrole Nanoarrays for Bio-Molecular Recognition
}

\author{
Patrícia Lisboa ${ }^{1}$, Marie-Bernadette Villiers ${ }^{2}$, Carine Brakha ${ }^{2}$, Patrice N. Marche ${ }^{2}$, \\ Andrea Valsesia ${ }^{3}$, Pascal Colpo ${ }^{*}{ }_{1}$ and François Rossi ${ }^{1}$
}

1 JRC-European Commission, IHCP, NBS, TP203, Via E. Fermi, 2749, 21027 Ispra (VA), Italy

2 INSERM U823 Université J. Fourier, Institut Albert Bonniot, BP170 38042 Grenoble Cedex 9,

France

3 Plasmore SRL Via G. Deledda, 4 - 21020 Ranco (Varese), Italy

*Address correspondence to this author at the JRC-European Commission,vIHCP, NBS, TP203, Via E. Fermi, 2749, 21027 Ispra (VA), Italy;Tel: +39033278997; Fax: +390332785787;

E-mail: pascal.colpo@jrc.ec.europa.eu

\begin{abstract}
The present study demonstrates that nanosphere lithography and electropolymerization can be successfully combined to produce bioactive protein nanoarrays. In particular, we describe a method to produce well-defined nanoarrays of polypyrrole functionalized with biomolecules. The nanoarrayed surfaces were fabricated on gold coated surface plasmon resonance prisms by first creating silicon oxide or polyethylene oxide nanotemplate using nanosphere lithography. The nanotemplate was subsequently used to grow bio-functionalized polypyrrole nanoarrays by electrocopolymerization. Atomic force microscopy analysis showed that the fabricated surfaces have a wellorganized 2D hexagonal geometry with nanoscale dimensions. The biological activity of the bio-functionalized polypyrrole was assessed by surface plasmon resonance detection. The results showed that the immobilized biomolecules within the nanoarrayed polypyrrole films had the necessary bioactivity for successful molecular recognition. Moreover the detection signals normalized to the bioactive area were increased by a factor 5 as compared to non-structured bio-functionalized polypyrrole in the nanoarrayed surfaces using polyethylene oxide.
\end{abstract}

Keywords: Colloidal lithography, immunoreaction, nanoarrays, polypyrrole, SPR. 


\section{INTRODUCTION}

The improvement of the performance of label-free biosensors is fundamental to expand their potential use in applications such as environment monitoring, food quality assessment and medical diagnosis [1]. The optimization of quality factors such as sensitivity, specificity and limit of detection are essential to attain this objective. One of the fundamental aspects is related to the sensor "bio-interface"

i.e. the location where the biomolecules interact with the transducer surface and where the biomolecular recognition occurs. The main challenge resides in the design of optimized biointerfaces that allow the immobilization of the bioreceptors in an active state supporting efficient biomolecular recognition i.e. promoting high affinity and specific detector/analyte reaction, thus guarantying a good detection performance.

A current approach to improve specific recognition is based on the functionalization of surfaces with chemical nanocontrasts to promote the selective immobilization of the bio-detector on bioadhesive nanoareas [2-5]. It has been shown that such systems favour a limited steric hindrance and a preferential antibody orientation resulting in a sensing performance improvement. Furthermore, nanoarrayed surfaces can present special optical and electrochemical properties that enhance the detection [6,7]. A variety of approaches are available to create nanoarrayed surfaces [8]. Among them, nanosphere lithography presents many advantages such as easiness of implementation, flexibility and cost effectiveness [9]. This technique has been applied to create nanocontrasts of self-assembled monolayers, plasma polymers and conductive polymers $[4,5,10,11]$.

The use of electrochemical polymerization of conductive polymers to functionalize surfaces represents a potentially efficient method [12]. One of the most important features of the electrochemical deposition resides in the relative simplicity to incorporate biomolecules into the polymer film. Adding biomolecules to the electrolyte solution during electrochemical deposition or using monomers modified with biomolecules allows the physical entrapment of the bioreceptor in the growing film [1-3 17]. Commonly conductive polymers have been applied in electrochemical transducer fabrication [18-20] but lately polypyrrole (PPy) and polyaniline have been used for optical detection [14, 18, 21]. PPy is a material of choice for bio-analytical sensors because of its good environmental stability and excellent biocompatibility along with the possibility of being substituted with relevant bio-functional groups [14]. Combining micro-arraying technology and electropolymerisation of biomolecule containing PPy has been successfully developed for producing biosensing systems [22, 23].

The present study demonstrates that nanosphere lithography and electro-polymerization can be combined to produce bio active protein nanoarrays. The first step to produce the protein nanoarrays is the creation of gold nanoelectrodes distributed in an insulating silicon oxide (SiOx) matrix. The nanoelectrodes are subsequently used for the electrochemical growth of PPy functionalized with biomolecule probes creating protein nanoarrays. The biofunctionalized PPy nanoarrays were used as platforms for monitoring antigen/antibody interactions by using Surface Plasmon Resonance imaging (SPRi). The influence of a nonfouling background on the detection performance was assessed by depositing a plasma polymerized polyethylene oxide (PEO) film on the SiOx matrix. The results were compared to those obtained with uniformly functionalized PPy micro-spots. 


\section{MATERIALS AND METHODS}

\subsection{Nanotemplate Fabrication}

The gold/SiOx nanotemplates were fabricated on a goldcoated glass prism (Genoptics France) following a procedure already described elsewhere [24] and illustrated in Fig. (1). Briefly, the fabrication consisted in the creation of a $2 \mathrm{D}$ hexagonal crystalline monolayer of selfassembled polystyrene nanobeads (nominal diameter $500 \mathrm{~nm}$ ) (Sigma- Aldrich) on the gold surface by spin coating. An oxygen plasma etching was then performed to reduce the bead diameter defining the size of the nanomask. Afterwards, an insulating SiOx layer was deposited through the nanomask by Plasma Enhanced Chemical Vapour Deposition to form the nanotemplates. Finally the beads were removed in water/ultrasounds. To create the passivated background, the SiOx coated surface was covered by a $10 \mathrm{~nm}$ layer PEO deposited in a homemade plasma reactor using a mixture of diethylene glycol monomethyl ether (Sigma-Aldrich) and argon [25].

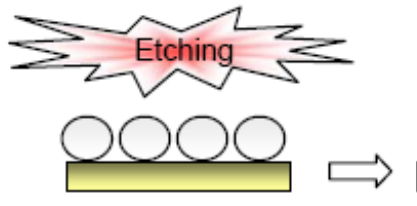

(a)

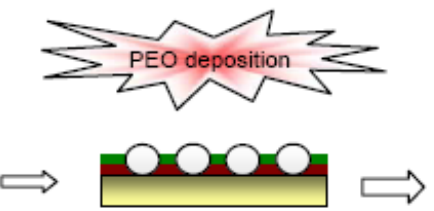

$\left(c^{3}\right)$

(d)

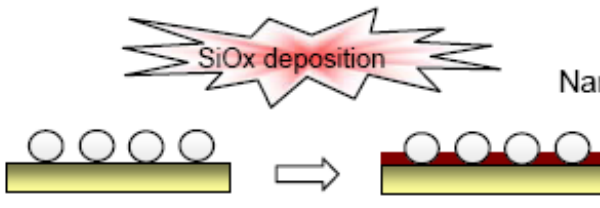

(b)
Nanobeads lift-off

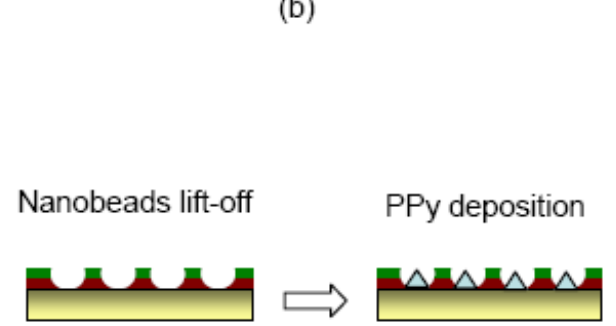

(e)
Nanobeads lift-off

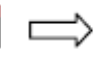

(c)

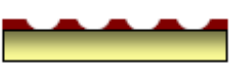

(d)

PPy deposition

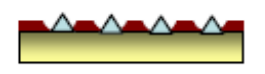

Fig. (1). Scheme of the production of the gold/SiOx nanotemplate (a) Plasma etching of the PS beads (b) PE-CVD deposition of SiOx layer, (c') PE-CVD deposition of PEO layer, (c) and (d) liftoff of the beads, (d') and (e) polypyrrole deposition by electro-co-polymerisation.

\subsection{PPy Array Fabrication}

The electrochemical deposition was performed using a micro-arrayer (pipette tip including a platinum wire used as a counter electrode on the SPR chip) by applying a short electrical pulse (2 Volts for $100 \mathrm{~ms}$ ) as previously described [14]. The bio-functionalized PPy was obtained by electrochemical copolymerization of pyrrole and pyrrole modified with biomolecules. PPy was grown either modified with Ovalbumin or with a-casein (prepared by using NHS functionalized pyrrole, INSERM, Grenoble, France). The unmodified PPy was obtained by simple pyrrole electropolymerization. The electrochemical deposition was done directly on the flat gold layer of the SPR chip or through the nanotemplate (SiOx or PEO) created on the gold layer, creating respectively uniform micro-spots or nanoarrayed micro-spots of PPy.

This procedure originated three types of nanoarrayed spots:

- Ova nanoarrays (probe)

- Casein nanoarrays (control)

- PPy nanoarrays (control)

And, three types of uniform spots:

- Ova uniform (probe)

- Casein uniform (control)

- PPy uniform (control) 


\subsection{SPRi Experiments}

The SPRi measurements were performed with SPRi- LAB+ instrument (GenOptics-HORIBAScientific, France) equipped with a laser source with a wavelength of $810 \mathrm{~nm}$. The reflectivity curves (or plasmon curves) were analyzed for all the different spots in order to determine the optimal working angle. The SPR measurements started by flowing the running $10 \mathrm{mM}$ phosphate buffer solution (PBS) at $\mathrm{pH} 7.4$, with 40 I/ $/ \mathrm{min}$ of flow rate until obtaining a stable signal that was used as the initial base line. The biomolecule solutions were prepared in PBS and injected with the same flow rate via a 500_I loop into the cell chamber. In the case of the uniform PPy microspots and the nanoarrays based on SiOx/PPy, the first step of the SPRi measurement consisted in blocking the surface with a Bovine serum albumin (BSA) solution (1\% in PBS) during 10 min. Unbound BSA molecules were then removed by injecting first PBS $(10 \mathrm{~min})$, then Glycine $\mathrm{HCl}$ $(0.1 \mathrm{M} \mathrm{pH} 2.3$, for $10 \mathrm{~min})$ followed by an injection of Tris Buffer (20mM, pH9.6) with Urea ( $4 \mathrm{mM})$ and EDTA $(20 \mathrm{mM})$ during $5 \mathrm{~min}$. In the case of the nanoarrays based on SiOx-PEO/PPy the recognition of the immobilized molecules was performed directly without blocking. The recognition step was carried out with rabbit polyclonal anti-Ova immunoglobulin (Rockland) [23] injected at different concentrations. After each recognition step, the chip was regenerated with Glycine $\mathrm{HCl}$ and Urea (as done after BSA blocking). The regeneration with these solutions was successful, bringing the SPR signal to the initial base line and giving the possibility to reuse the sensor and to inject various concentrations using the same chip successively.

\section{4 .Atomic Force Microscopy (AFM) Measurements}

Scanning probe microscopy experiments were performed with a commercial AFM (SMENA head, Solver electronics from NT-MDT). The AFM characterization of the samples was performed in tapping mode in ambient air by using silicon AFM probes (NSG 11 from NT-MDT) with stiff cantilevers (nominal force constant of $5.5 \mathrm{~N} / \mathrm{m}$ ) and sharpened conical tips (nominal curvature radius of $10 \mathrm{~nm}$ ). The AFM probe was driven to oscillate at resonance frequency (around $150 \mathrm{kHz}$ ). A tapping ratio between setpoint oscillation amplitude (fixed at about $100 \mathrm{~nm}$ ) and free oscillation amplitude of about 0.7 has been used.

\section{RESULTS AND DISCUSSION}

\subsection{PPy Nanoarray Fabrication and Characterization}

AFM analysis has been performed in order to characterize the topography of the PPy nanoarrays. AFM images of SiOx nanotemplates are presented in Fig. (2). As already described in a previous publication [11], the deposition of the PS nanobeads by spin coating results in a 2D hexagonal crystalline bead arrangement. The AFM images of SiOx nanotemplates evidence that the initial hexagonal fingerprint of the nanobeads is kept after the plasma etching, showing that this operation did not modify their initial arrangement (Fig. (2a) and (2b)). The cross section of the fabricated nanoholes is characterized by a conical shape with the bottom area smaller than the upper one due to the isotropic character of the SiOx deposition (Fig. (2e) and (2f)).

The bottom and the upper side dimensions of the truncated cone are dependant on the matrix thickness (SiOx and PEO film thickness). The sizes of the nanoholes for both configurations are summarized in Table 1. With these dimensions, the total gold nanoareas represent $8 \%$ and $6 \%$ of the total surface area for SiOx and PEO-covered SiOx matrix, respectively. The contrast of the two materials was confirmed by the phase image (Fig. (2c) and (2d)), evidencing the presence of spots with a different phase located at the centre of the holes. Such an evident phase contrast is due to the presence of gold only at the central part of the nanoholes, while SiOx or PEO layer cover the surrounding area. 

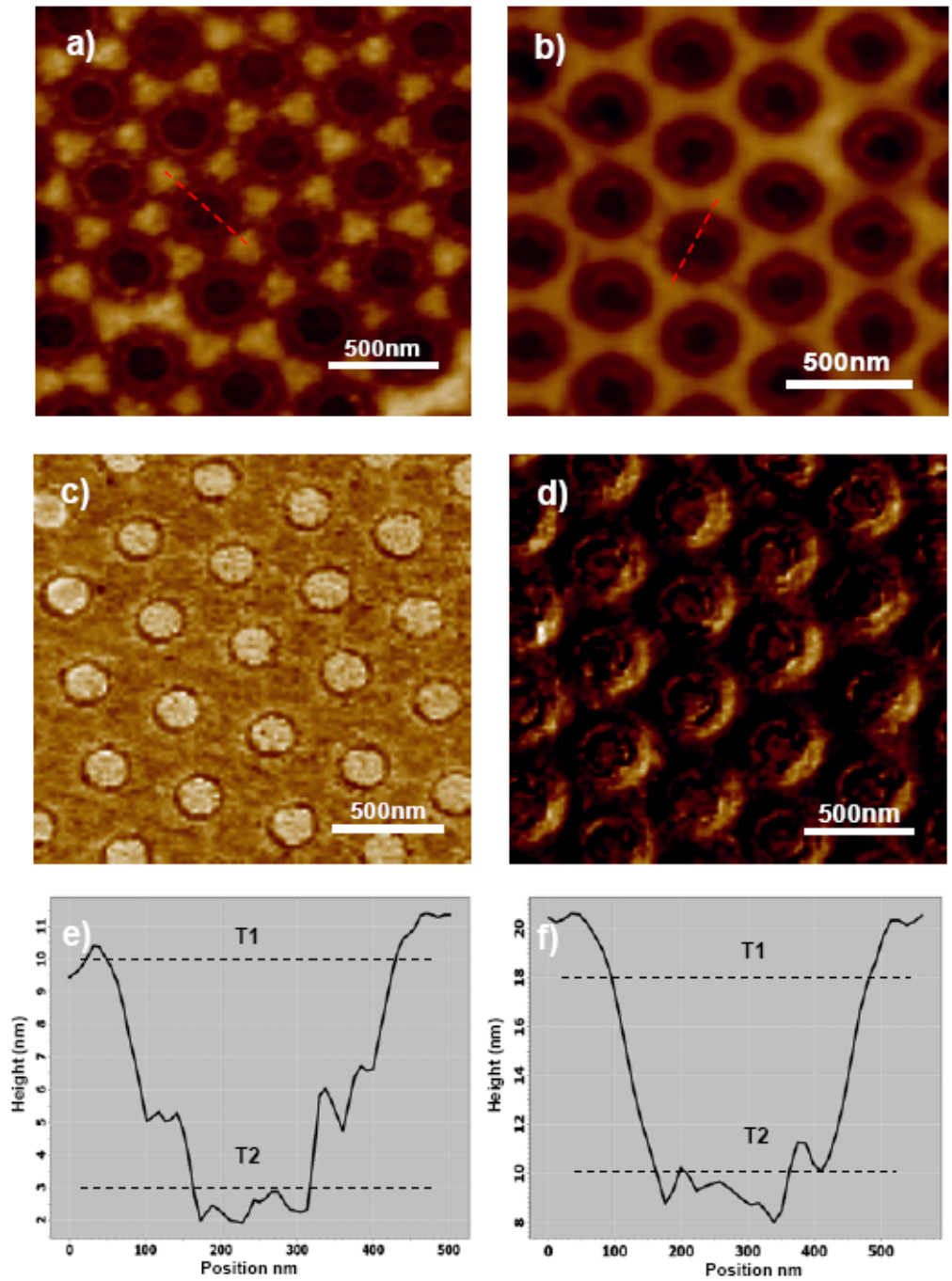

Fig. (2). AFM analysis of the nanotemplates: a-c-e) $10 \mathrm{~nm} \mathrm{SiOx}$ and b-d-f) $10 \mathrm{~nm} \mathrm{SiOx}+10 \mathrm{~nm}$ of PEO; a-b) topographic images, $\mathbf{c}-\mathbf{d}$ ) corresponding phase images and e-f) height profiles along the dot lines. [a) vertical scale $0-20 \mathrm{~nm}$; c) color scale: -80 to $10 \mathrm{o}$; b) vertical scale $0-25 \mathrm{~nm}$; d) color scale: -10 to 0 o].

Table 1. Truncated Nanohole Dimension

\begin{tabular}{|c|c|c|}
\hline & SiOx nanoarrays & PEO passivated nanoarrays \\
\hline $\mathrm{H}(\mathrm{nm})$ & $117 \pm 1.2$ & $22.4 \pm 1.9$ \\
\hline Upper diameter $(\mathrm{nm})$ & $363 \pm 14.9$ & $359 \pm 51.9$ \\
\hline Bottom diameter $(\mathrm{nm})$ & $179 \pm 10.6$ & $155.6 \pm 22.6$ \\
\hline
\end{tabular}

Uniform PPy films functionalized or not by biomolecules (OVA, casein) were electro-spotted on the bare gold surface creating spots 400 to $500 \mathrm{~m}$ of diameter with $10 \mathrm{~nm}$ of thickness. The same spotting parameters were used to grow the $\bar{P} \bar{P} y$ through the SiOx nanotemplates (Fig. (3)). The functionalized PPy growth occurs only within the gold nanoareas where the electron transfer 
takes place, creating a ring-like geometry with height of about $10 \mathrm{~nm}$, as shown in Fig. (3d). This result shows that SiOx layer of $10 \mathrm{~nm}$ was sufficient to act as insulating layer. The ring-like geometry probably results in a lower PPy area as compared to the total gold surface available in the nanoholes. This ring-shape can be explained by the fact that in potentiostatic electrocopolymerization, the same electrical potential conditions can produce smooth or spikeshape films depending on the nanoholes, representing less than $10 \%$ of the uniform surface area, results a local surface charge, increased by a factor larger than 10 as compared to a uniform configuration. This can explain why, on a uniform gold surface, the films are homogenous whereas in the nanoholes the films have a nonuniform cylindrical shape. These electrochemical deposition conditions have been chosen because these parameters are known not to damage the biomolecules grafted on the PPy [14, 21, 22, 27].
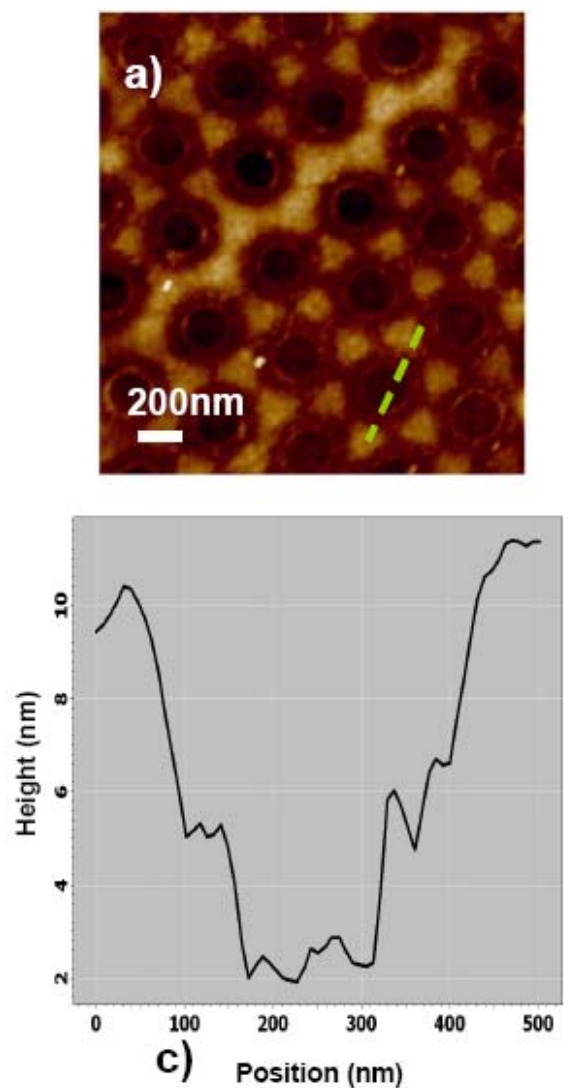
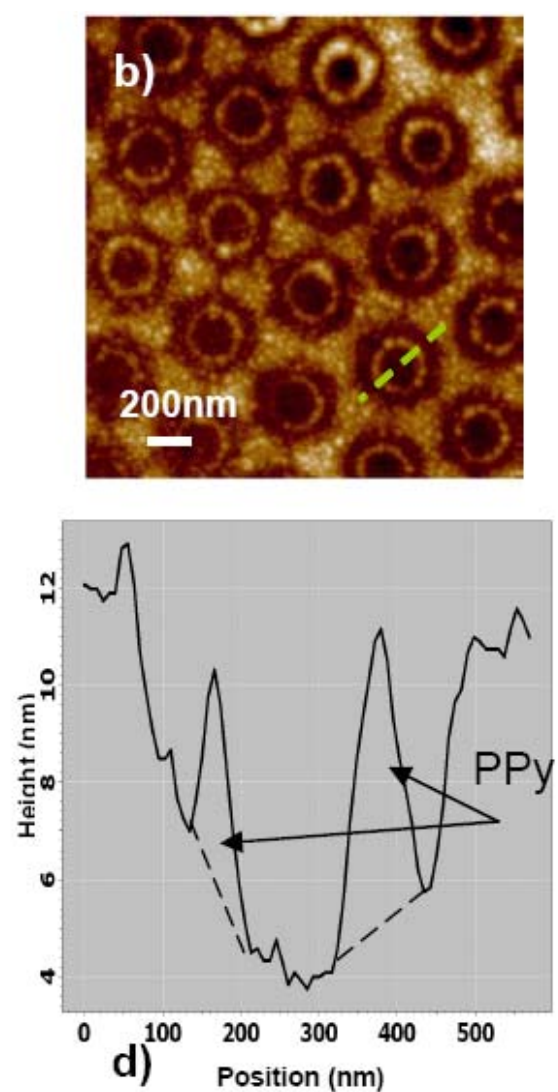

Fig. (3). AFM images and profiles comparing the SiOx nanotemplate nanoarrays before (a-c) and after (b-d) the PPy growth. a-c) topographic images, b-d) height profiles along the dot lines. (c)-(d) vertical scale $0-20 \mathrm{~nm}$.

\subsection{SPRi Studies}

The reactivity of the bio-functionalized nanoarrayed PPy was assessed by using SPR $i$ detection. The assays were based on the interaction between the probes immobilized on the surface and their specific antibody. As mentioned above, two configurations were studied SiOx/PPy or PEO/PPy. In the first configuration a blocking step with BSA was performed to cover the SiOx areas avoiding unspecific binding. In both configurations the reflectivity curves obtained with the nanoarrayed surfaces presented a less pronounced dip of reflectivity as compared to those obtained with uniform PPy (data not shown). The SPRi sensitivity is directly 
dependant on the slope of the plasmon curves, thus this low reflectivity dip affects negatively the performance of the nanoarrayed surfaces.

It should be notice that the bioactive area (area containing the probes) in the nanoarrayed surfaces was less than $10 \%$ of the bioactive area in the uniform surfaces. If the probes have the same bio-activity in the different surfaces, the detection signals obtained with nanoarrayed surfaces should be proportional to the bio active area i.e. should be only $10 \%$ of the signal obtained with the uniform surfaces.

The original SPR sensorgrams (variation of the reflectivity upon ligand binding) measured for some of the antibody concentrations are shown in supplementary information.

\subsubsection{Proteins Nanoarrays with SiOx Background}

In these experiments, before the bio-interactions the SiOx matrix was blocked with BSA in order to reduce unspecific binding. The nonspecific adsorption (background signal) was determined by injecting anti-Ova on unmodified PPy or PPy modified with the non specific probe (casein). The resulting background signal was lower than $10-15 \%$ of the specific signals for all the control surfaces showing a good specificity of detection for both uniform and nanoarrayed surfaces. Fig. (4). presents the SPR signals as a function of the Anti-Ova concentration after background subtraction. The signals increase with the concentration of anti-OVA. The specific signals from nanoarrayed PPy surface were much lower than the ones obtained on the uniform surface. The SPR signals of OVA nanoarrays represent $15-20 \%$ of the OVA uniforms films signal, which is higher than the expected in case of similar bio-activity of the probes. These results show that the OVA embedded within the nanoarrayed PPy maintain its bioactivity allowing anti-OVA biorecognition, and slightly increases the bio-activity of the probes.

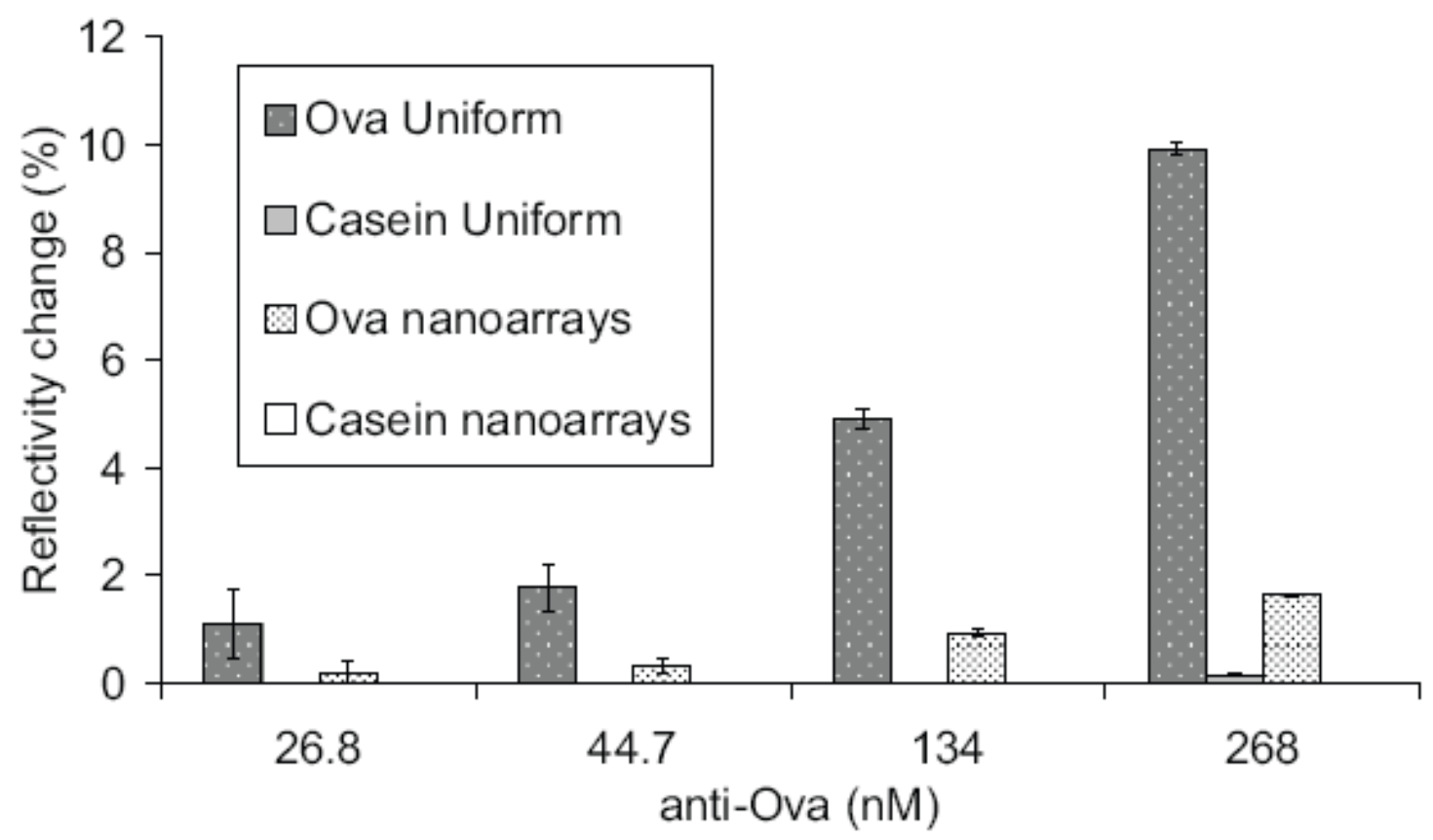

Fig. (4). SPRi signals related with the bio-interactions on SiOx nanoarrayed and uniform PPy surfaces. Different concentrations of anti-Ova were successively injected on a SPR chip grafted with Ova (assay), Casein (control). 


\subsubsection{Protein Nanoarrays with PEO Background}

Plasma polymerized PEO has already shown high potential to reduce protein adhesion [28]. In this work, plasma deposited PEO films were used to passivate the SiOx background and therefore avoid the blocking step by BSA injection. After PEO deposition (10 nm) on the surface of the chip, the PPy electro-copolymerization was performed through the PEO coated nanotemplate. Functionalized and unmodified PPy were used. The original SPR sensorgrams obtained in this type of surfaces (for some of the antibody concentrations) are shown in supplementary information. The background signal obtained upon Anti-Ova injection was lower for both the nanoarrayed and the uniform surface $(<10 \%$ of the unmodified PPy and casein surfaces) showing that PEO layer limited the non-specific adsorption on the nanoarrayed surfaces. An interesting result is the fact that as compared to SiOx matrix signal (Fig. (5)), leading to $55-65 \%$ of the uniform surface signals. In addition, taking into account that the nanoarrayed surfaces present only $6 \%$ of the active area of uniform surfaces, the nanoarrayed surface recorded signal normalized by the bioactive area is increased by a factor 5 as compared to the uniformly functionalized surfaces.

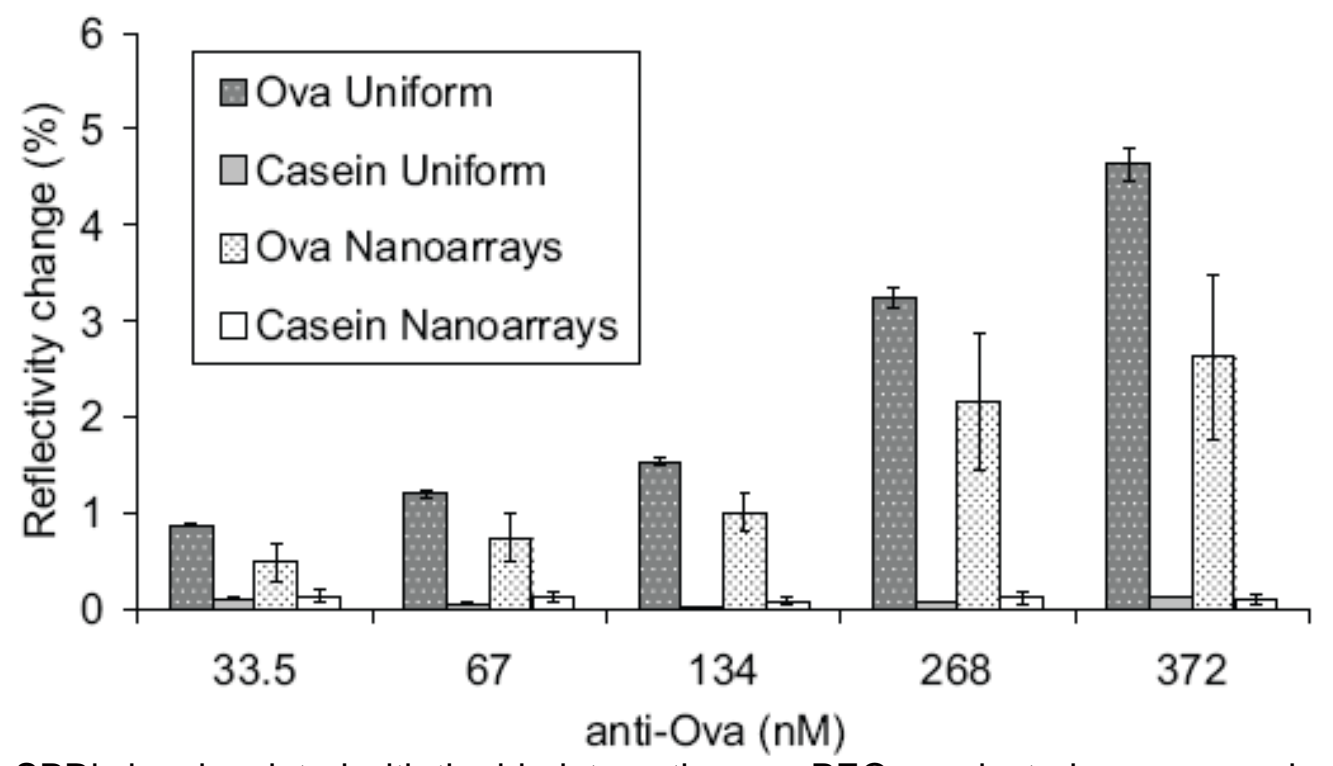

Fig. (5). SPRi signals related with the bio-interactions on PEO passivated nanoarrayed and uniform PPy surfaces. Different concentrations of anti-Ova were successively injected on a chip grafted with Ova (assay), Casein (control).

These results show that the use of PEO as passivating layer avoids blocking step with BSA and improves the biorecognition signal as compared to non-passivated matrix configuration. $A$ possible explanation to this improvement can be the fact that the presence of BSA obstruct OVA accessibility to the antibodies in the nanoareas. The signal increase could also be a consequence of the interaction of the surface plasmon wave with the optical grating created by the refractive index contrast of the different materials constituting the nanoarrayed surface. This effect has been already observed for another type of nanoarrayed self assembled monolayers [10] showing SPR signal improvement as compared to the control uniform surface one and to a non-ordered nanoarrayed surfaces. In our case, the optical configuration is more complex (Fig. 6) and the combination of the refractive index seems to be less favourable (see Table 1 supplementary information). Nevertheless, replacing the BSA monolayer (refractive index $=$ 
1.55) by a $10 \mathrm{~nm}$ PEO film highly hydrated i.e. with a lower refractive index, increases the refractive index contrast favouring optical grating effects. Nevertheless, at this stage, it is difficult to determine if the signal improvement is due to better binding site accessibility or to an optical amplification. Studies are ongoing to help elucidating these results.
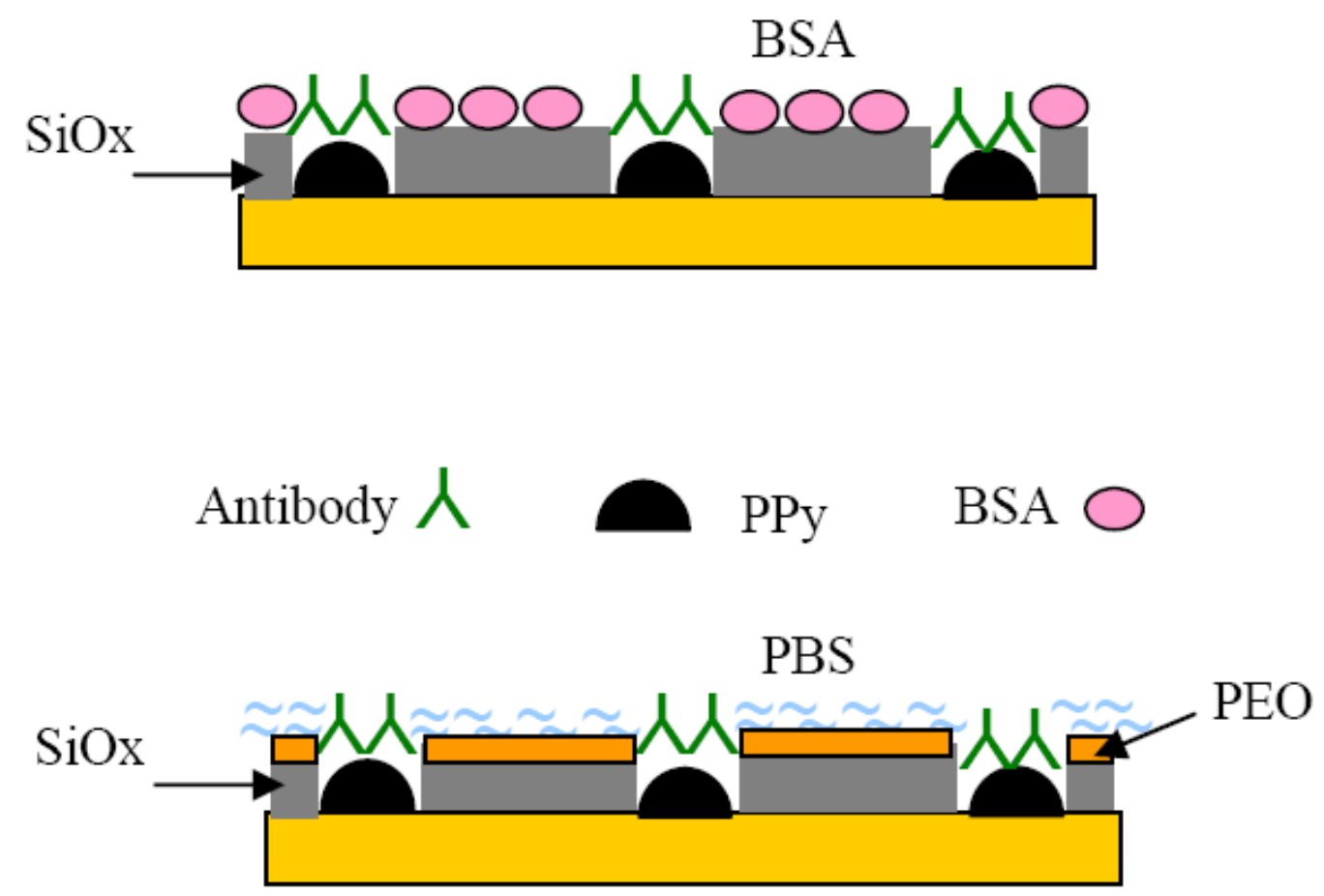

Fig. (6). Schema of the distribution of the biomolecules on the nanoarrayed surface without and with PEO layer during the bio-interaction.

\section{CONCLUSIONS}

The combination of nanosphere lithography and electrocopolymerization showed to be a successful method to develop surfaces with well-organized geometry and nanoscale dimension. The PPy nanoarrays developed in this work showed to be suitable for biomolecular interaction analysis by SPRi detection. The nanoarrays modified with proteins provided a good signal of detection and background noise at different analyte concentrations, which was improved with the passivation of the SiOx matrix with PEO layer. This indicates that proteins immobilized via electrochemical polymerization of PPy-protein conjugates on nanoarrays presented good binding site availability enabling their recognition by their corresponding ligand. The combination of PPy nanoarrays with PEO treatment appears promising to design bio-detectors useful for efficient protein analysis.

\section{ACKNOWLEDGMENT}

The authors acknowledge FP6 Nano2Life mobility program for the exchange grant between ISERM (Grenoble, France) and JRC (Ispra, Italy).

\section{SUPPLEMENTARY MATERIAL}

Supplementary material is available on the publishers Web site along with the published article : Micro and Nanosystems, 2011, 3, 83-89 


\section{REFERENCES}

[1] Luong, J.; Male, K.; Glennon, J. Biosensor technology: Technology push versus market pull. J. Biotech. Adv., 2008, 26, 492-500.

[2] Frederix, F.; Bonroy, K.; Laureyn, W.; Reekmans, G.; Campitelli, A.; Dehaen, W.; Maes, G. Stability of mixed PEO_thiol SAMs for biosensing applications. Langmuir, 2003, 19(10), 4351 4357.

[3] Valsesia, A.; Colpo, P.; Meziani, T.; Lisboa, P.; Lejeune, M.; Rossi, F. Protein: nanopatterns for improved immunodetection sensitivity. Langmuir, 2006, 22(94), 1763-1767.

[4] Agheli, H.; Malmström, J.; Larsson, E. M.; Textor, M.; Sutherland, D. S. Large area protein nanopatterning for biological applications. Nano Lett., 2006, 6(6), 1165-1171.

[5] Krishnamoorthy, S.; Himmelhaus, M. Confinement induced enhancement of antigen-antibody interactions within binary nanopatterns to achieve higher efficiency of on-chip immunosensors.

Adv. Mat., 2008, 14(20), 2782-2788.

[6] Barnes, W. L.; Dereux, A.; Ebbesen, T. W. Surface plasmon subwavelength optics. Nature, 2003, 424, 824-830.

[7] Arrigan, D. W. M. Nanoelectrodes, nanoelectrode arrays and their applications. Analyst, 2004, 129(12), 1157-1165.

[8] Christman, K.L.; Enriquez-Rios, V. D.; Maynard, H. D. Nanopatterning proteins and peptides. Soft Matter, 2006, 2, 928- 939.

[9] Yang, S. M.; Jang, S. G.; Choi, D. G.; Kim, S.; Yu, H. K. Nanomachining by colloidal lithography. Small, 2006, 2(4), 458- 475.

[10] Lisboa, P.; Valsesia, A.: Mannelli, I.; Mornet, S.; Colpo, P.; Rossi, F. Sensitivity enhancement of surface-plasmon resonance imaging by nanoarrayed organothiols. Adv. Mat., 2008, 20(12), 2352-2358.

[11] Valsesia, A.; Colpo, P.; Mannelli, I.; Mornet, S.; Bretagnol, F.; Ceccone, G.; Rossi, F. Protein nanopatterns for improved immunodetection sensitivity. Anal. Chem., 2008, 80(19), 73367340.

[12] Ramanavi_ius, A.; Ramanavi_iene, A.; Malinauskas, A. Electrochemical sensors based on conducting polymer-polypyrrole. Electrochim. Acta, 2006, 51(27), 6025-6037.

[13] Haddour, N.; Cosnier, S.; Gondran, C. Electrogeneration of a poly(pyrrole)-NTA chelator film for a reversible oriented immobilization of histidine-tagged proteins. J. Am. Chem. Soc., 2005, 127(16), 5752-5753.

[14] Grosjean, L.; Cherif, B.; Mercey, E.; Roget, A.; Levy, Y.; Marche, P. N.; Villiers, M. B.; Livache, T. A. Polypyrrole protein microarray for antibody-antigen interaction studies using a labelfree detection process. Anal. Biochem., 2005, 347(2), 193-200.

[15] Reiter, S.; Habermüller, K.; Schuhmann, W. A reagentless glucose biosensor based on glucose oxidase entrapped into osmiumcomplex modified polypyrrole films. Sens. Act. B, 2001, 79, 150-156.

[16] Yasuzawa, M.; Matsuki, T.; Mitsui, H.; Kunugi, A.; Nakaya, T. Properties of glucose sensors based on the immobilization of glucose oxidase in N-substituted polypyrrole film. Sens. Act. B, 2000, 66, 77-79.

[17] Chaubey, A.; Pande, K.; Singh, V. S.; Malhotra, B. D. Coimmobilization of lactate oxidase and lactate dehydrogenase on conducting polyaniline films. Anal. Chim. Acta, 2000, 407, 97-103. [18] Lange, U.; Roznyatovskaya, N. V.; Mirsky, V. M. Conducting polymers in sensors and arrays. Anal. Chim. Acta, 2008, 614, 1-26.

[19] Gerard, M.; Chaubey, A.; Malhotra, B. D. Application of conducting polymers to biosensors. Biosens. Bioelec., 2002, 17(5), 345-359.

[20] Guimard, N. K.; Gomez, N. Schmidt, C. E. Conducting polymers in biomedical engineering. Prog. Polym. Sci., 2007, 32, 876-921. 
[21] Guedon, P.; Livache, T.; Martin, F.; Lesbre, F.; Roget, A.; Bidan, G.; Levy, Y. Characterization and optimization of a real-time, parallel, label-free, polypyrrole-based DNA sensor by surface plasmon resonance imaging. Anal. Chem., 2000, 71, 6003-6009.

[22] Livache, T.; Bazin, H.; Caillat, P.; Roget, A. Electroconducting polymers for the construction of DNA or peptide arrays on silicon chips. Biosen. Bioelec., 1998, 13, 629-634.

[23] Cherif, B.; Roget, A.; Villiers, C. L.; Calemczuk, R.; Leroy, V.; Marche, P. N.; Livache, T.; Villiers, M. B. Clinically related protein-peptide interactions monitored in real time on novel peptide chips by surface plasmon resonance imaging. Clin. Chem., 2006, 52(2), 255-262.

[24] Valsesia, A.; Lisboa, P.; Colpo, P.; Rossi, F. Fabrication of polypyrrole-based nanoelectrode arrays by colloidal lithography. Anal. Chem., 2006, 78(21), 7588-7591.

[25] Bretagnol, F.; Lejeune, M.; Papadopoulou-Bouraoui, A.; Hasiwa, M.; Rauscher, H.; Ceccone, G.; Colpo, P.; Rossi, F. Fouling and non-fouling surfaces produced by plasma polymerization of ethylene oxide monomer. Acta Biomater., 2006, 2, 165-172.

[26] Tietje-Girault, J.; Ponce de León, C.; Walsh, F. C. Electrochemically deposited polypyrrole films and their characterization. Surf. Coat. Tech., 2007, 201, 6025-6034.

[27] Livache, T.; Guedon, P.; Brakha, C.; Roget, A.; Levy, Y.; Bidan, G. Polypyrrole electrospotting for the construction of oligonucleotide arrays compatible with a surface plasmon resonance hybridization detection. Synth. Metals, 2001, 121(1-3), 1443-1444.

[28] Lopez, G.P.; Ratner, B.D.; Tidwell, C.D.; Haycox, C.L.; Rapoza, R.J.; Horbett, T.A. Glow discharge plasma deposition of tetraethylene glycol dimethyl ether for fouling resistant biomaterial. J. Biomed. Mat. Res., 1992, 26(4), 415-439. 